\title{
Trying to find a cure for cystic fibrosis: CFTR biomarkers as outcomes
}

\author{
To the Editor:
}

In a recent issue of the European Respiratory Journal, DE BOECK et al. [1] provided a systematic review of the clinimetric properties of cystic fibrosis transmembrane conductance regulator (CFTR) biomarkers, giving supporting evidence for promoting these biomarkers as surrogate end-points. The authors are to be congratulated not only for having collected exhaustive data about CFTR biomarkers and attempted to clarify their role but also for having led tremendous efforts towards standardisation of these techniques over recent years. They concluded that available data demonstrate reliability, validity and responsiveness of nasal potential difference (NPD), as well as validity and responsiveness of sweat chloride levels. However, one may wonder whether these conclusions are in agreement with the results of the last randomised clinical trials involving the three most promising small molecules in the context of searching for a CFTR mutationspecific treatment. A phase 3 randomised clinical trial evaluating the effects of ataluren has been recently completed [2]. The study involved 238 patients carrying at least one class I CFTR mutation who were randomised to receive ataluren or placebo for 48 weeks. From a pragmatic point of view, statistically significant benefit in terms of forced expiratory volume in $1 \mathrm{~s}$ (FEV1) was restricted to patients who were not using inhaled tobramycin, and the magnitude of the benefit was comparable to that reported with inhaled tobramycin [3]. The pulmonary exacerbation rate was $23 \%$ lower in favour of ataluren. In keeping with a previous study, sweat chloride levels were unchanged. In contrast to the three much smaller previous studies, epithelial chloride secretion as assessed by NPD did not improve. This raises several possibilities. If ataluren is an effective drug, the validity of NPD and sweat chloride as CFTR biomarkers may be questioned; if ineffective, the reliability of NPD may be doubted. Furthermore, in patients homozygous for the F508del mutation, lumacaftor alone $[4,5]$ or in combination with ivacaftor [5] induced similar, modest $\left(<10 \mathrm{mmol} \cdot \mathrm{L}^{-1}\right)$ but significant decreases in sweat chloride. However, improvement of FEV1 was only observed when combining the drugs. Again, this challenges the above conclusions, casting doubt on the validity of the biomarkers and raising the possibility that FEV1 improvement in these studies might be unrelated to ion transport correction.

-

@ERSpublications

CFTR biomarkers as surrogate end-points: recent trials cast doubt on validity of NPD and sweat chloride measurements http://ow.ly/nuTOD

Patrick Lebecque

Cliniques Universitaires St Luc, CF Unit, Brussels, Belgium.

Correspondence: P. Lebecque, Cliniques Universitaires St Luc, 10 Avenue Hippocrate, 1200 Brussels, Belgium. E-mail: Patrick_Lebecque@hotmail.com

Received: Nov 052012 | Accepted after revision: Jan 282013

Conflict of interest: None declared.

References

1 De Boeck K, Kent L, Davies J, et al. CFTR biomarkers: time for promotion to surrogate end-point? Eur Respir J 2013; 41: 203-216.

2 Konstan M, Accurso F, De Boeck K, et al. Targeting class 1 mutations: update on ataluren as a promising treatment for nonsense mutation CF. Pediatr Pulmonol 2012; 47: Suppl. 35, 108-109.

3 Ramsey BW, Pepe MS, Quan JM, et al. Intermittent administration of inhaled tobramycin in patients with cystic. fibrosis. Cystic Fibrosis Inhaled Tobramycin Study Group. N Engl J Med 1999; 340: 23-30.

4 Clancy JP, Rowe SM, Accurso FJ, et al. Results of a phase IIa study of VX-809, an investigational CFTR corrector compound, in subjects with cystic fibrosis homozygous for the F508del-CFTR mutation. Thorax 2012; 67: 12-18.

5 Boyle MP, Bell S, Konstan M, et al. The investigational CFTR-corrector, VX-809 (lumacaftor) co-administered with the oral potentiator ivacaftor improved CFTR and lung function in F508del homozygous patients: phase II study results. Pediatr Pulmonol 2012; 47: Suppl. 35, 315. 